\title{
Ophthalmic adverse drug reactions to systemic drugs: a systematic review
}

\author{
Ana Miguel $^{1,2 *}$, Filipe Henriques ${ }^{2}$, Luís Filipe Azevedo ${ }^{1}$ and Altamiro Costa Pereira ${ }^{1}$ \\ ${ }^{1}$ Center for Research in Health Technologies and Information Systems (CINTESIS) and Department of Health Information and Decision \\ Sciences, Faculty of Medicine, University of Porto, Portugal \\ ${ }^{2}$ Department of Ophthalmology, Central University Hospital of Coimbra, Coimbra, Portugal
}

\begin{abstract}
Purpose To perform a comprehensive and systematic review regarding ophthalmic adverse drug reactions (ADRs) to systemic drugs to: (i) systematically summarize existing evidence, (ii) identify areas, ophthalmic ADRs or drugs that lacked systematization or assessment (namely drugs with original studies characterizing specific ophthalmic ADRs but without causality assessment nor without meta-analysis).

Methods Systematic review of several electronic databases (last search 1/7/2012): Medline, SCOPUS, ISI web of knowledge, ISI Conference Proceedings, International Pharmaceutical Abstracts and Google scholar. Search query included: eye, ocular, ophthalmic, ophthalmology, adverse and reaction. Inclusion criteria were: (i) Primary purpose was to assess an ophthalmic ADR to a systemic medication; (ii) Patient evaluation performed by an ophthalmologist; (iii) Studies that specified diagnostic criteria for an ocular ADR. Different types of studies were included and analyzed separately. Two independent reviewers assessed eligibility criteria, extracted data and evaluated risk of bias.

Results From 562 studies found, 32 were included (1 systematic review to sildenafil, 11 narrative reviews, 1 trial, 1 prospective study, 6 transversal studies, 6 spontaneous reports and 6 case series). Drugs frequently involved included amiodarone, sildenafil, hydroxychloroquine and biphosphonates. Frequent ophthalmic ADRs included: keratopathy, dry eye and retinopathy.

Conclusions To increase evidence about ophthalmic ADRs, there is a need for performing specific systematic reviews, applying strictly the World Health Organization's (WHO) definition of ADR and WHO causality assessment of ADRs.

Some ophthalmic ADRs may be frequent, but require ophthalmological examination; therefore, ophthalmologists' education and protocols of collaboration between other specialties whenever they prescribe high-risk drugs are suggestions for the future. Copyright $\left({ }^{\circ} 2014\right.$ John Wiley \& Sons, Ltd.
\end{abstract}

KEY WORDS—adverse drug reactions; clinical pharmacology; pharmacoepidemiology; ophthalmology

Received 13 March 2013; Revised 10 November 2013; Accepted 9 December 2013

\section{INTRODUCTION}

Ophthalmology is perhaps one of the medical specialties in which there are the fewest assessed adverse drug reactions (ADRs), representing a particular challenge in Pharmacovigilance. ${ }^{1}$ However, the eye is a complex organ in which minimal impairment can produce a substantial functional effect. ${ }^{2}$ Ophthalmic ADRs are usually not continuously detected, although they might be either frequent or specific of a drug or drug group, such as acute angle-closure glaucoma and myopic shift caused by topiramate, ${ }^{3}$ cataracts provoked by

*Correspondence to: A. Miguel, Department of Health Information and Decision Science, Faculty of Medicine of Oporto. Rua Quinta do Sardoal, VE3, n¹0, 4430-182 V.N.Gaia, Portugal. Email: myworld_ana@hotmail.com corticosteroids, ${ }^{4}$ floppy iris syndrome caused by tamsulosine $^{5}$ and uveitis caused by rifabutin. ${ }^{6}$

Some ADRs are rare but can cause irreversible blindness (such as in optic atrophy provoked by ethambutol), ${ }^{7}$ while others are extremely frequent but usually harmful (namely cornea verticillata caused by amiodarone) ${ }^{8}$

There are reports that suggest ophthalmic ADRs provoked by a systemic drug, but remain unsupported because no systematic review has been performed. Many ophthalmic ADRs have been identified solely due to spontaneous reports, namely blurred vision caused by leuprolide, ${ }^{9}$ or other ophthalmic ADRs caused by different drugs such as biphosphonates, cetirizine or isotretinoin. ${ }^{2}$ Therefore, on the one hand, prospective studies or trials should be performed to study the causality of each drug to each ophthalmic ADR; on the other hand, a systematic review should 
be performed to clarify and assess what ophthalmic ADRs can occur after the correct prescription of each drug. A systematic review would be useful not only to identify drugs in which ophthalmic ADRs are frequent or serious, but also to increase knowledge of physicians (prescribing physicians and ophthalmologists), enabling a greater detection of ophthalmic ADRs after an ophthalmic examination and enabling the construction of multi-disciplinary protocols whenever a high-risk drug is prescribed.

General ADRs are estimated to cause of $2.7 \%$ to $15.7 \%$ hospital admissions and to occur in $16.9 \%$ of hospitalized patients (confidence interval 95\%: $13.6 \%, 20.2 \%) .{ }^{11}$ In a study performed in the United States (US), it was estimated that more than 100000 people die every year as a consequence of fatal ADRs, placing fatal ADRs between the fourth and sixth leading causes of death in the US. ${ }^{12}$ However, the specific frequency of ophthalmic ADRs is not known.

Although there are several studies about ophthalmic ADRs, this theme presents with specific difficulties, ${ }^{13,14}$ and the methods of identification and reporting ADRs vary greatly. ${ }^{15,16}$ Some studies have established recommendations, ${ }^{17,18}$ and others offer guidelines to performing systematic reviews of studies of ophthalmic ADRs. ${ }^{19,20}$

We intended to perform a general systematic review about ophthalmic ADRs to systemic drugs in order to, on the one hand, systematically summarize existing evidence, and on the other hand, identify areas of specific ophthalmic ADRs or drugs that lacked systematization or assessment. This includes the identification of drugs that cause specific ophthalmic ADRs which are well described in original studies but without systematic review nor meta-analysis (therefore, opportunities for specific systematic reviews with metaanalysis in the future are also identified).

\section{METHODS}

We performed a systematic review of studies that assessed ophthalmic ADRs to systemic drugs according to the guidelines of the Cochrane Collaboration ${ }^{19}$ and PRISMA Statement, ${ }^{21}$ adapted to this theme.

\section{Definitions}

We used the following definition for adverse drug reaction: "any noxious, unintended and undesired effect of a drug, which occurs at doses used in humans for prophylaxis, diagnosis, or therapy", according to the World Health Organization (WHO) definition ${ }^{22}$ of 1972.
An adverse event is: "an injury related to medical management, in contrast to complications of disease". ${ }^{23}$ Medical management includes all aspects of care, including diagnosis and treatment, failure to diagnose or treat and the systems and equipment used to deliver care. ${ }^{23}$ Therefore, to increase specificity, we wanted to assess only adverse drug reactions.

\section{Search methods}

We searched through several electronic databases (last date of search was 1/7/2012): Medline, SCOPUS, ISI web of knowledge, ISI Conference Proceedings, International Pharmaceutical Abstracts and Google scholar. We used a search query created after a pilot study to add specificity (full search query available if requested to the corresponding author) that included the terms: eye, ocular, ophthalmic, ophthalmology, adverse and reaction. We searched for grey literature and unpublished data and hand-searched all references of included studies and relevant reviews.

\section{Selection criteria}

Studies were included if they followed all inclusion criteria listed below:

(1) Studies in which the primary purpose was to assess an ophthalmic ADR to a systemic medication. Since there is a wide misuse of the terms ADR, adverse event (AE) and adverse drug event (ADE), we obtained also the full text of studies who claimed to assess AEs or ADEs, to verify their methodology, and to include the studies that actually assessed ADRs, although they called it AEs or ADEs.

(2) Studies with patient evaluation performed by an ophthalmologist.

(3) Studies that specified diagnostic criteria for an ocular ADR.

We also included studies with different languages (we hired a translator), any country and experimental studies (if any). We did so to have a more thorough and complete literature search. We did not exclude systematic nor narrative reviews if they added useful information about ocular ADRs, as we intended to have a general overview that summarized and added further systematization to existing evidence, and to identify areas or specific ophthalmic ADRs that lacked systematization or assessment.

\section{Exclusion criteria:}

(1) Studies assessing adverse events that did not correspond to ADRs (for example, we excluded 
reports of capsular rupture in phacoemulsification surgery, but we did not exclude reports of capsular rupture due to intra-operatory floppy iris syndrome, a syndrome that is an ADR provoked by tamsulosine or other drugs).

(2) Systemic ADRs to topical ophthalmic drugs, or ophthalmic ADRs to topical ophthalmic drugs (they were not the purpose of our study and would increase heterogeneity and reduce clarity of our study).

(3) Studies that were comments or letters, if they would not add new scientific evidence to our review. However, letters or comments that included case reports not published elsewhere about specific ocular ADRs were not excluded, in order to identify rare ophthalmic ADRs.

(4) Studies assessing drugs already removed from the market.

\section{Data collection and extraction}

Two independent reviewers, AM and FH, first examined each title and abstract to exclude obviously irrelevant reports and then independently examined each full text report, to determine eligibility according to inclusion criteria. Disagreements were solved by consensus, recorded and analyzed using kappa statistics.

Primary outcome was the presence and type of ocular ADR and the respective causative systemic drug. Secondary outcomes included: ocular structure affected, diagnosis, serious or vision-threatening ADR. All symptoms, visual acuity (VA), signals and results of complementary examination performed at presentation were recorded, as well as after a follow-up. Attitude or treatment performed for each ADR was also registered (suspension of the causative drug, specific treatment, administration of an antidote, no treatment necessary). If VA was not recorded in the $\log$ MAR scale, ${ }^{24}$ it was converted.

We always assessed the drug name, identified the therapeutic drug class according to Anatomical Therapeutic Chemical Classification System of $\mathrm{WHO}^{25}$ and reported the number of days during which the drug was used and the administration route (if that information was available). We verified if causality was assessed in the original studies (and according to what classification, preferably WHO's ${ }^{23}$ or Naranjo's, ${ }^{26}$ and respective results) as well as predictability of ADRs (using Hartwig's predictability scale, for example), ${ }^{27}$ preventability (e.g. Schumok \& Thornton's preventability criteria) ${ }^{28}$ and types of ADRs (Rawlins and Thompson's classification ${ }^{29}$ ). We did not intend to identify all of the ophthalmic ADRs, but to systematize the most important and the most frequent ADRs according to the results of our systematic search.

\section{Risk of bias assessment}

We performed risk of bias assessment for each included study and recorded it in a standardized form created to assess ADR studies (in a previous work ${ }^{10}$ ) and adapted to Ophthalmology after a pilot study. We did not use scales (discouraged by the Cochrane approach $^{20}$ ) but criteria from Cochrane, STROBE, ${ }^{30}$ QUOROM $^{31}$ and PRISMA ${ }^{21}$ adapted to the particular scope of ophthalmic ADR evaluation, which included: complete description of study design, description of study type (case report, case series, prospective observational study, trial,...), adequate diagnostic criteria for ophthalmic ADR, complete ophthalmologic evaluation at presentation, quantified visual acuity at presentation and follow-up, results of complementary testing described at presentation and follow-up, definition of ADR presented, rationale for study size, causality assessment of ADR, preventability assessment of ADR, description of all statistical methods, characterization of study participants, description of methods to prevent bias (information bias, selection bias and other bias) and presentation of complete summary measures. The two reviewers independently assessed study quality and risk of bias; disagreements were solved by consensus.

Studies were divided in low risk of bias (5 or less parameters with medium, unclear or high risk of bias), medium risk (6 to 9 ) and high risk (10 or more parameters evaluated as medium, unclear or high risk of bias).

\section{RESULTS}

\section{Literature search}

Pubmed search yielded 124 results; SCOPUS yielded 72 results; Google Scholar 60; ISI Web of Knowledge yielded 154; others yielded 152. From these 562 studies (corresponding to 300 distinct studies), 163 were selected to obtain full text and then 32 studies were included $^{9,17,32-61}$ (Fig. 1): 1 systematic review of ADRs to a specific drug, 11 narrative reviews, 1 trial, 1 prospective study, 6 case-control or cohort or crosssectional studies, 6 spontaneous reports and 6 case reports or case series. Kappa agreement for study inclusion was 0.80 during the first phase and 0.82 during the full text review (good agreement).

\section{Characteristics of included studies}

Table 1 summarizes the characteristics of included studies. We identified several types of studies of ocular 


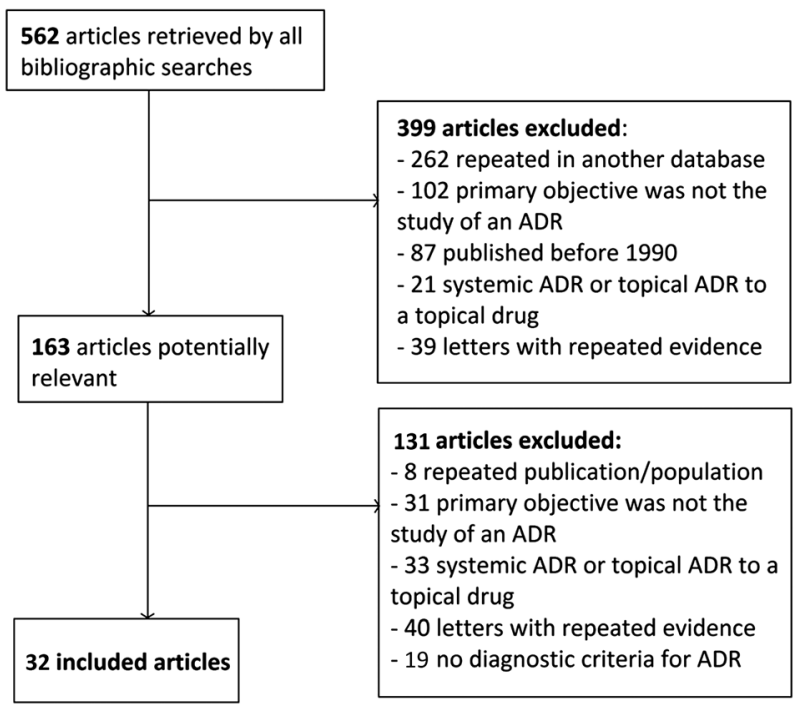

Figure 1. Flowchart of search strategy

ADRs, most of them narrative reviews without systematic criteria nor bibliographic search.

\section{Ophthalmic ADRs}

Many different ophthalmic ADRs exist to many systemic drugs. In Table 2, we represent a summary of the main ophthalmic ADRs found in this systematic review, according to each specific drug, dose, risk factors and tried to characterize the ophthalmic ADR (if reported) and to evaluate the level of evidence of each of the studies reporting each ADR (according to the Oxford Levels of Evidence ${ }^{62}$ ). Keratitis, retinopathy, glaucoma, dry eye and blurred vision were the most frequent ADRs identified.

We identified many ophthalmic ADRs to drugs that have original studies but are currently lacking a systematic review (therefore representing an opportunity for further studies, as described in the Discussion Section, below). Many studies were found but only one systematic review (of ophthalmic ADRs to sildenafil ${ }^{56}$ ) and few narrative reviews with systematic search were performed. Therefore, examples of drugs that cause ophthalmic ADRs that would benefit from a recent and specific systematic review are: tamoxifen, amiodarone, antidepressants, phenotiazines, hydroxychloroquine, oral contraceptives, etc.

\section{Risk of bias assessment}

In Fig. 2, we present the summary of our quality evaluation of included studies, according to each parameter assessed - risk of bias graph. Few studies had low risk of bias. Only one study performed rationale for study size. Most studies (25) performed a complete initial evaluation by an ophthalmologist, but only 11 performed a follow-up of at least 1 month. Only 13 studies performed causality assessment for ADR and only 7 applied or presented WHO's definition of an ADR. Risk of bias summary, which contains detailed risk of bias assessment for each included study, is available if requested to contact author.

\section{DISCUSSION}

\section{What this study adds}

There is an increasing number of studies of ophthalmic ADRs. In spite of the common belief that ADRs in Ophthalmology are rare, some ADRs might be extremely frequent (such as cornea verticillata caused by amiodarone ${ }^{8}$ ), but require specific ophthalmological examination for its detection. Every ocular structure might be affected by an ADR. There is a need for performing specific systematic reviews of ophthalmic ADRs, because the majority of included studies were narrative non-systematic reviews, most of which without the strict application of WHO's definition of ADR nor causality assessment of ADRs.

Several drugs that may provoke different ophthalmic ADRs were identified, namely amiodarone, sildenafil, psychotropic drugs, alpha-blockers, corticosteroids and topiramate. Although cornea verticillata is found very frequently in patients medicated with amiodarone (authors report a rate of $100 \%{ }^{46}$ ), this finding rarely reduces visual function; on the other hand, amiodarone may provoke a rare optic neuropathy that may provoke marked visual loss. ${ }^{18}$ Sildenafil and tadalafil have been recently studied, but while some authors report no difference between ERG patterns of placebo versus these drugs, ${ }^{53}$ others found several ADRs associated with sildenafil, ${ }^{58}$ namely: ischemic optic neuropathy, central retinal vein occlusion, cilio-retinal artery occlusion, acute angle closure glaucoma and optic atrophy.

Strengths of our systematic review lie in the comprehensive search performed, the general increase in systematization and characterization of ophthalmic ADRs, the summary of existing evidence according to WHO's causality criteria for ADR and WHO's definition of ADR and finally the identification of specific ophthalmic ADRs that could benefit from future specific systematic reviews with possible meta-analysis.

Limitations of our systematic review include not only heterogeneity found in different types of ADRs but also the extreme variability in the methodologies of studies of ophthalmic ADRs (from isolated case reports to retrospective series of spontaneous reports, 
OPHTHALMIC ADVERSE DRUG REACTIONS - SYSTEMATIC REVIEW
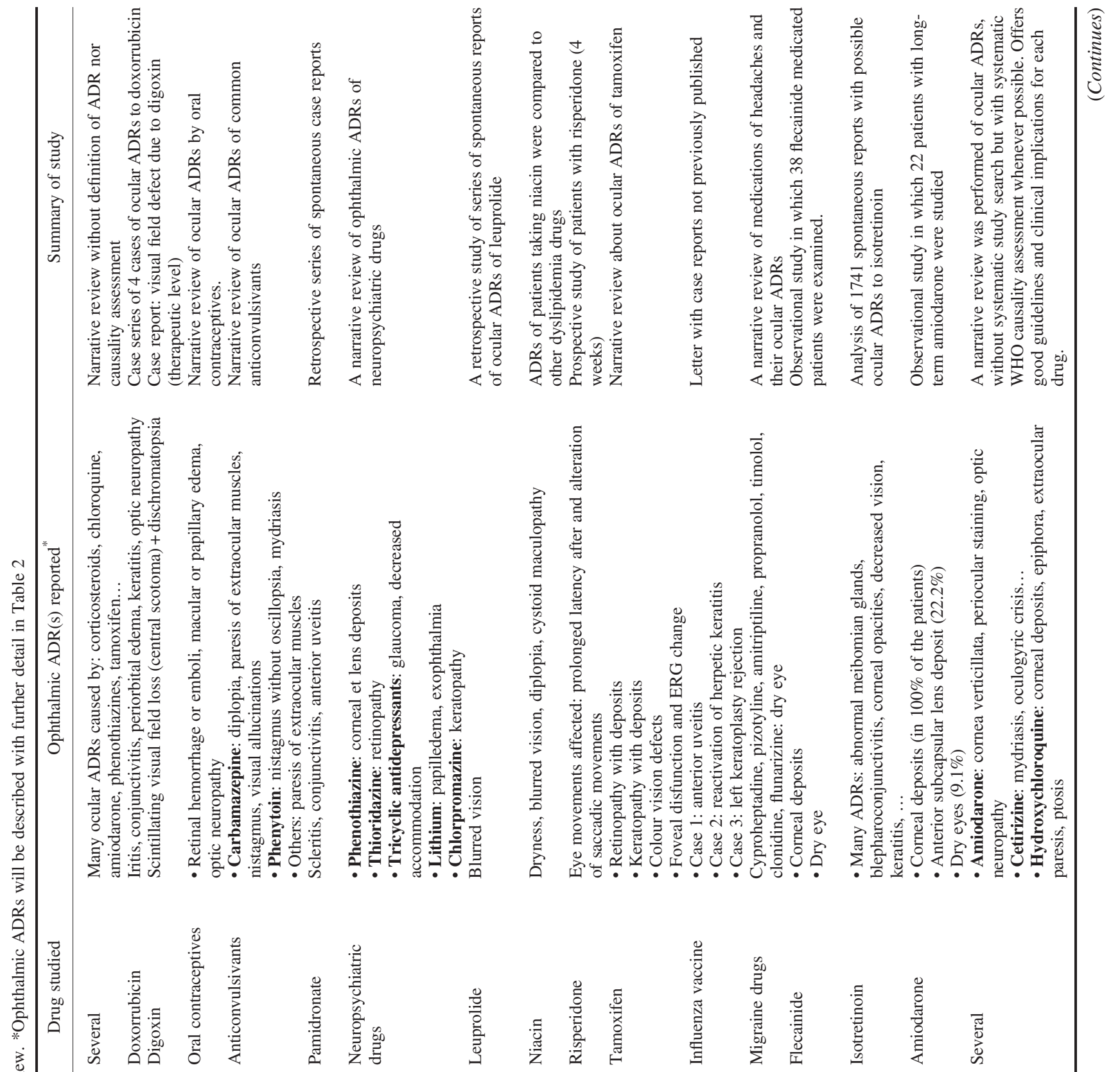

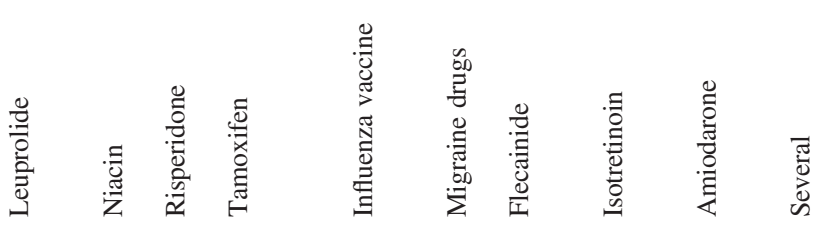

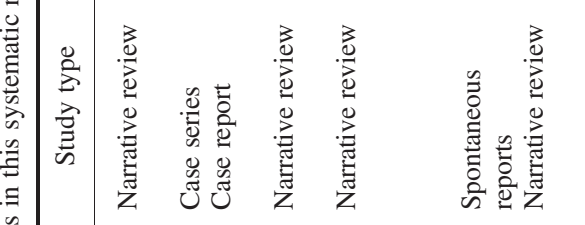

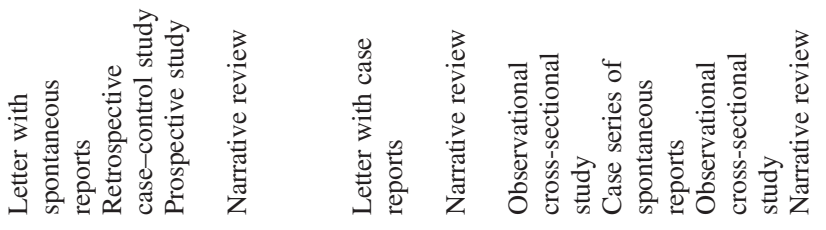

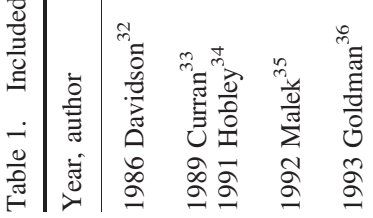

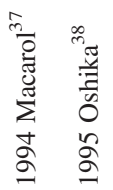

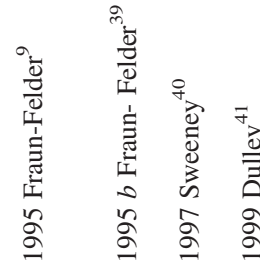

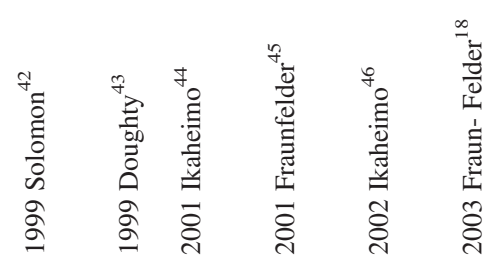


A. MIGUEL $E T A L$.
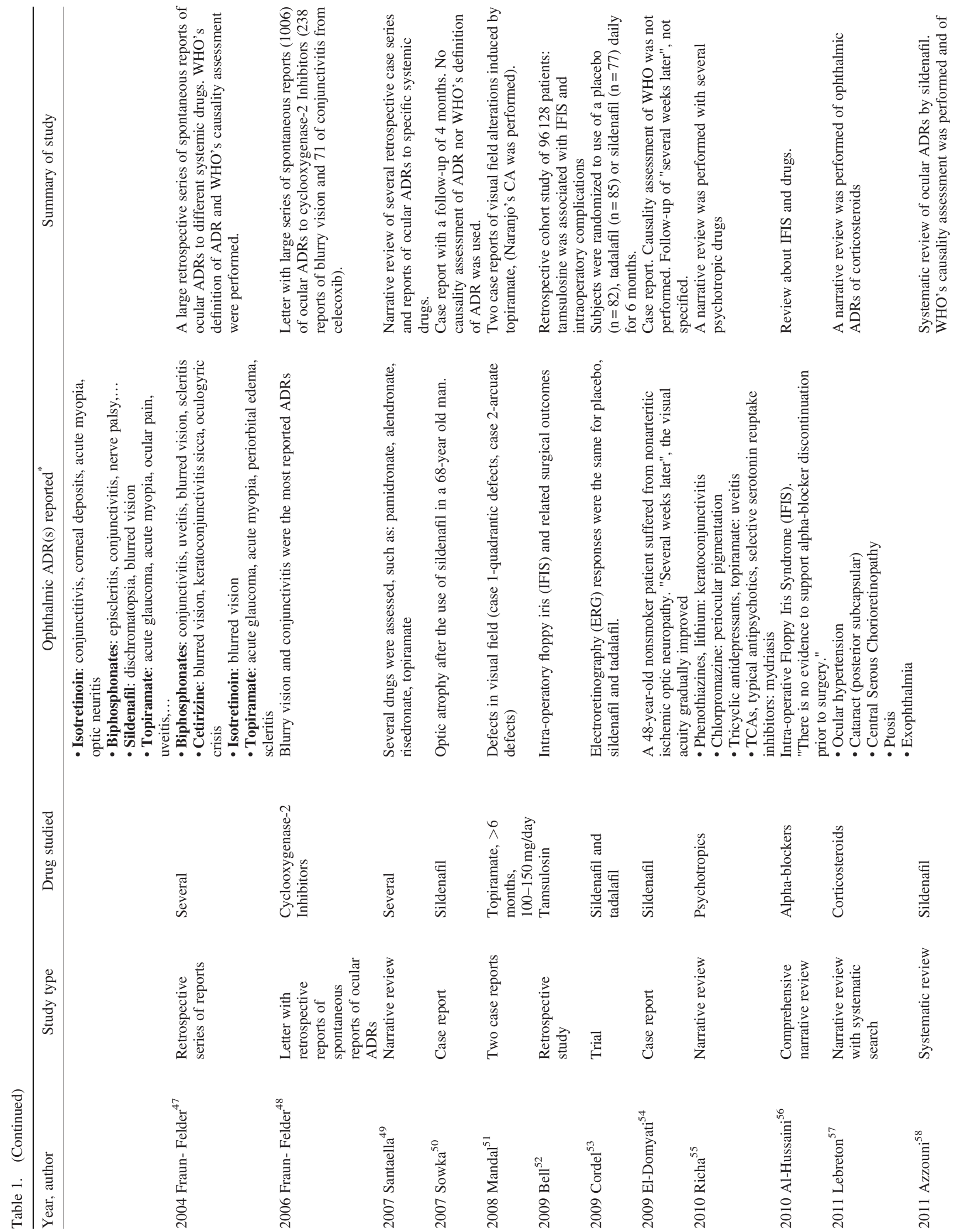

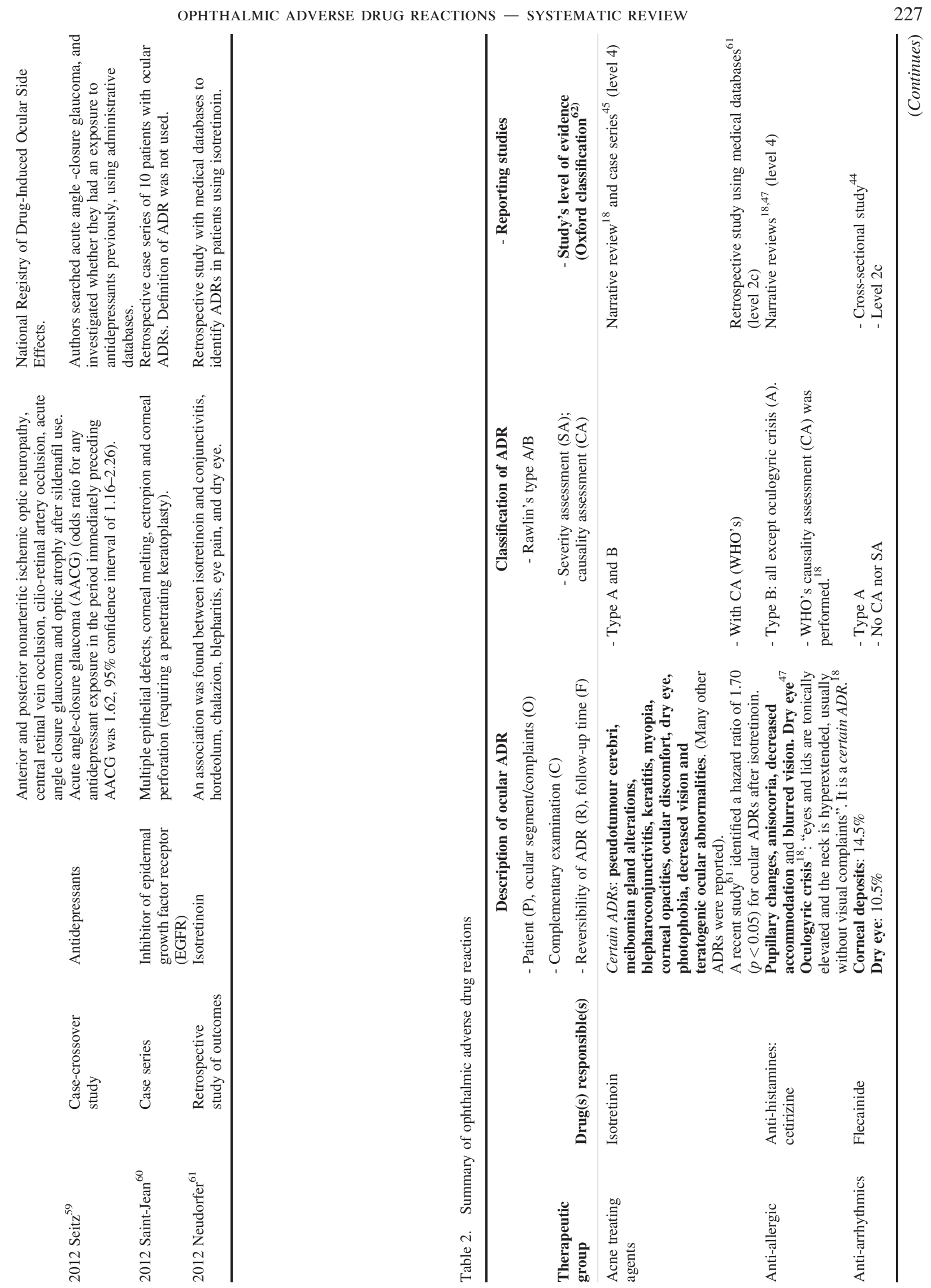
A. MIGUEL $E T A L$.

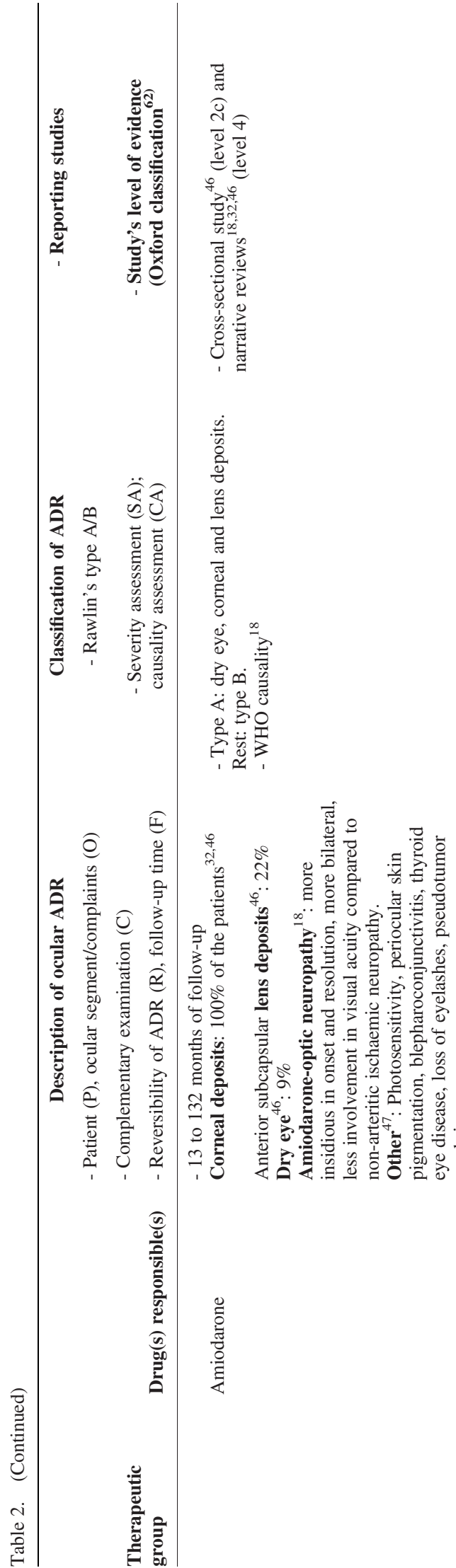

Copyright (C) 2014 John Wiley \& Sons, Ltd.

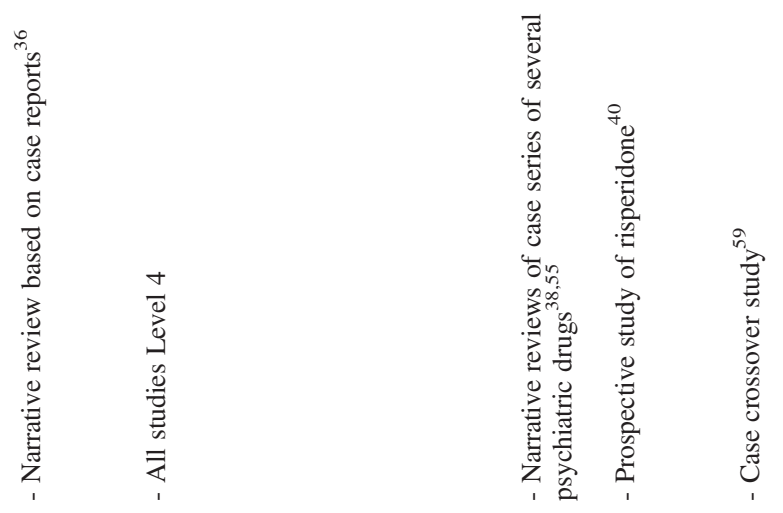

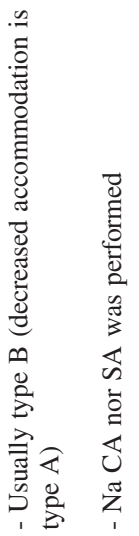
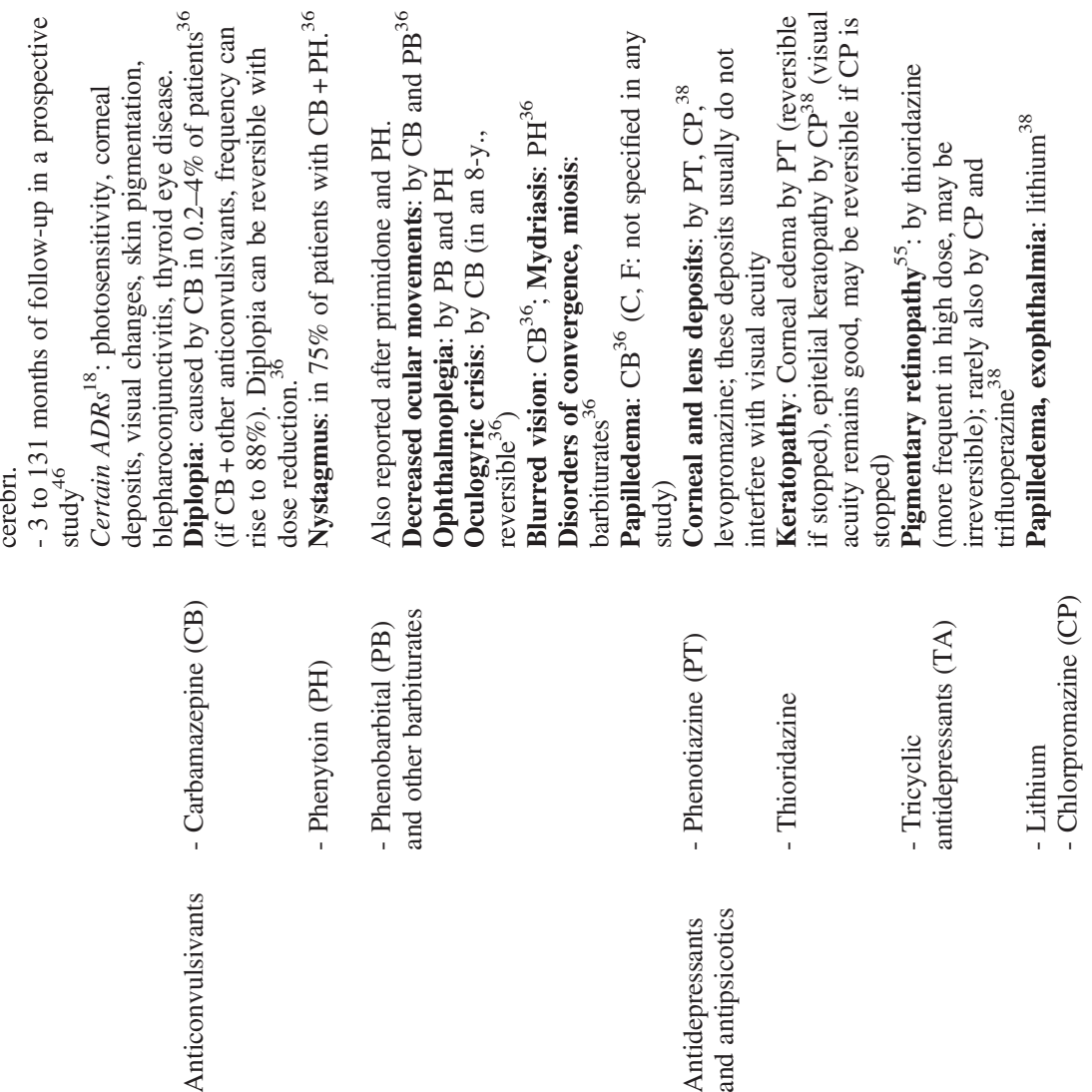


$$
\text { OPHTHALMIC ADVERSE DRUG REACTIONS — SYSTEMATIC REVIEW }
$$
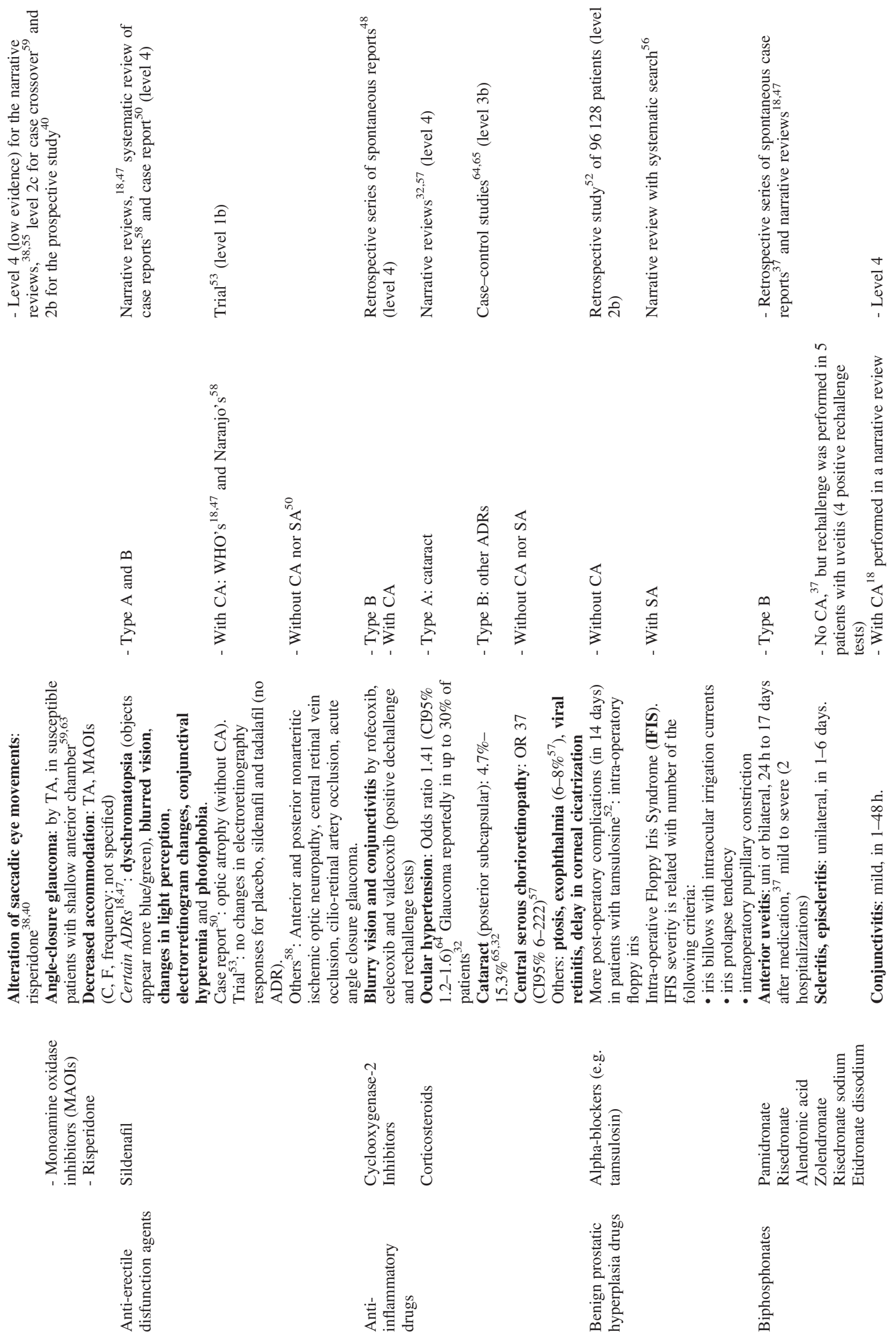

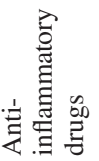
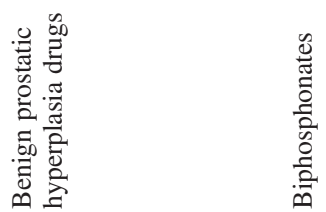
A. MIGUEL $E T A L$.

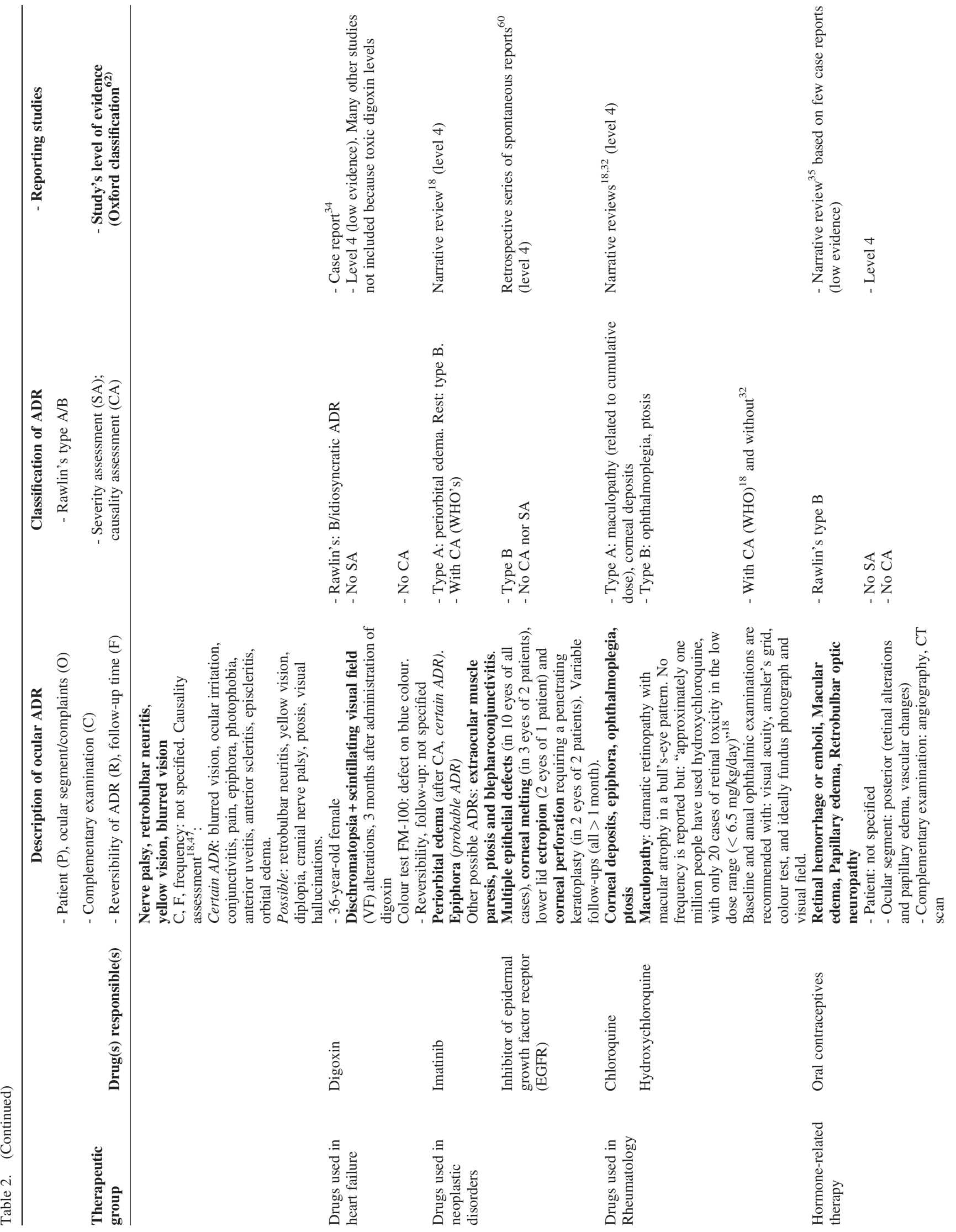



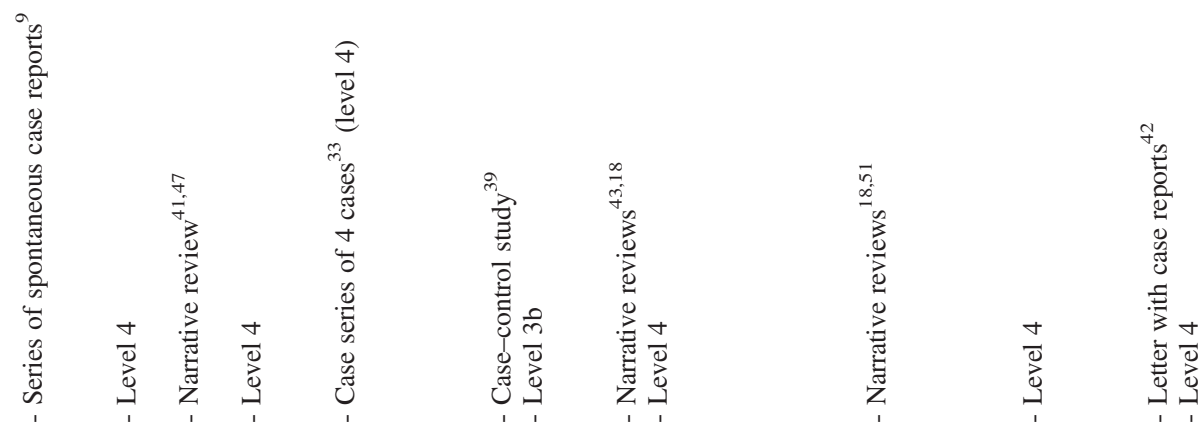

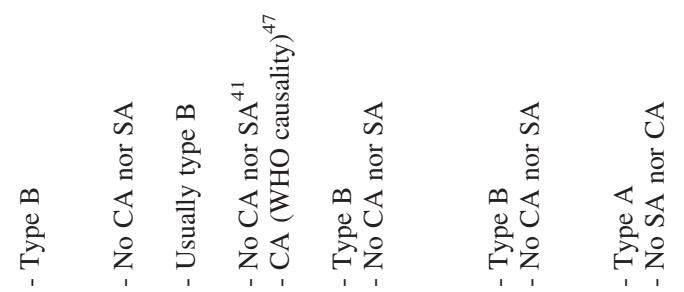
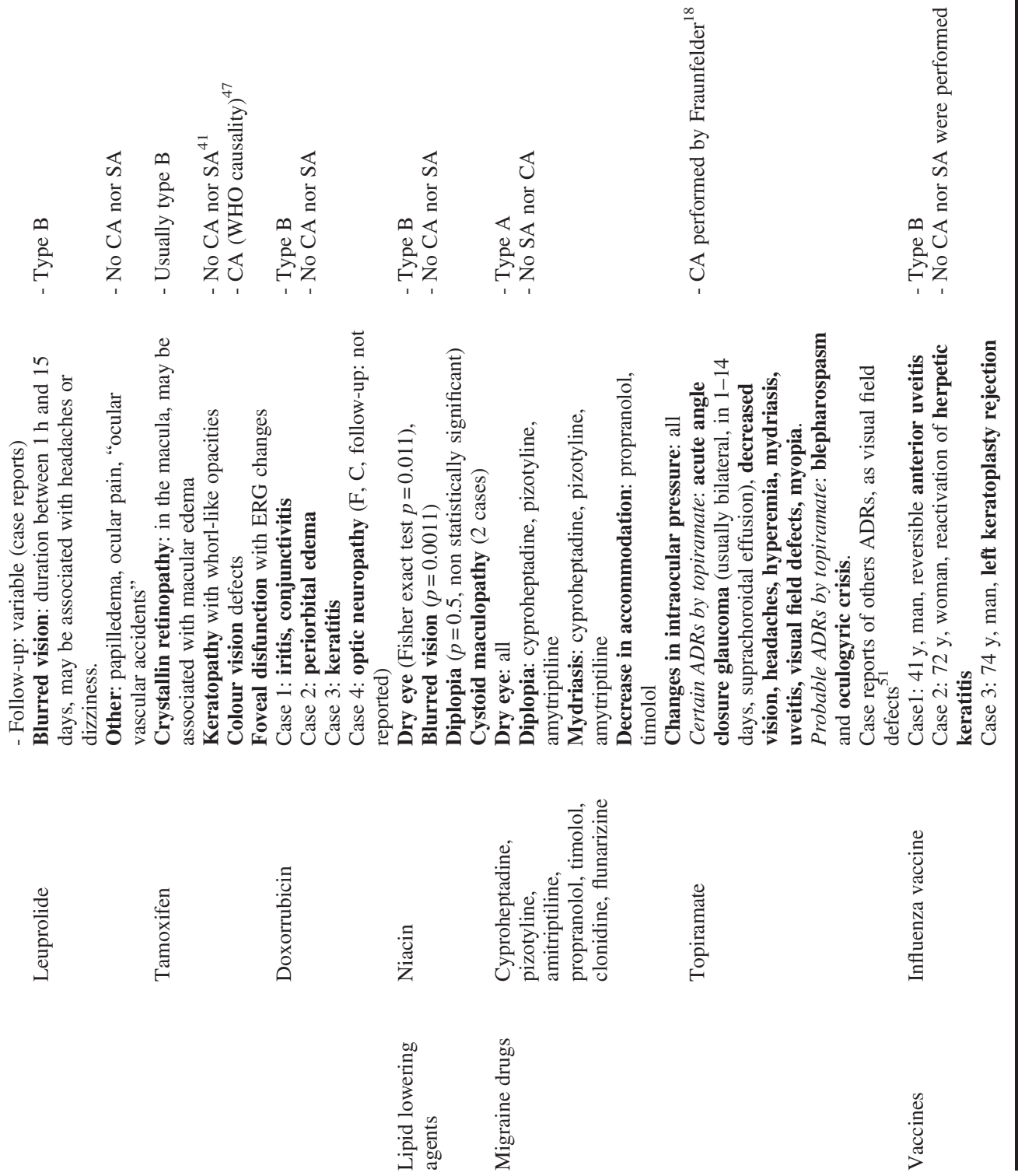


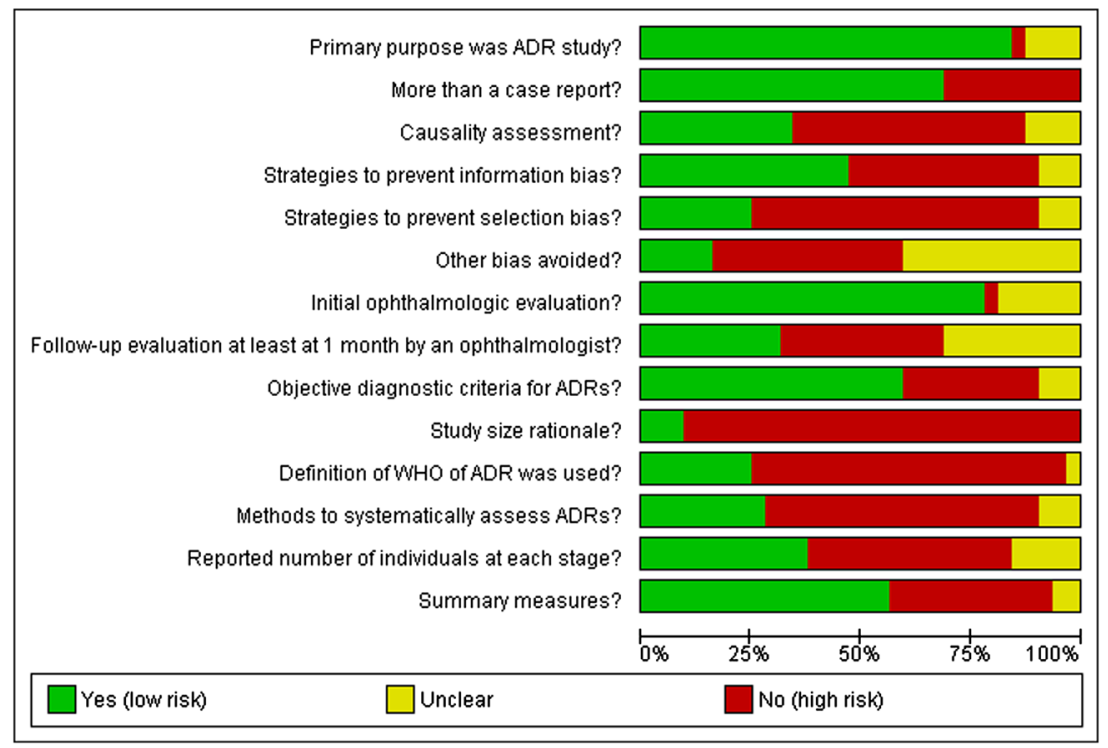

Figure 2. Risk of bias graph

prospective observational studies and trials). These limitations were expected, because this was a systematic review with a very general scope and because the detection of ophthalmic ADRs depends on the degree of suspicion and an adequately performed ophthalmologic examination. Many ophthalmic ADRs are only detected by case reports or spontaneous reports, representing a limitation but simultaneously an opportunity to improve. Consequently, there are many ophthalmic ADRs that are based on a low level of evidence. We believe this is an additional reason for applying systematically the WHO definition for ADR and a causality assessment (whether WHO's or Naranjo's), in order to decrease doubts. High-risk drugs such as the ones identified in Table 2 should be associated with protocols of evaluation (especially in susceptible individuals or in high doses) by an ophthalmologist, in order to detect sooner and with higher sensitivity and specificity the respective ophthalmic ADRs.

\section{CONCLUSION}

Ophthalmologists' education (to increase recognition of ophthalmic ADRs) and the dissemination of protocols of collaboration between Ophthalmology and other Medicine specialties whenever they prescribe high-risk drugs (such as sildenafil, biphosphonates, psychiatric medication, tamoxifen, hydroxichloroquine) are strong suggestions for the future.

\section{CONFLICT OF INTEREST}

The authors declare no conflict of interest.

\section{KEY POINTS}

- Ophthalmology is perhaps one of the medical specialties in which there are the few assessed ADRs, but the eye is a complex organ in which minimal impairment can produce a substantial functional effect.

- We performed a systematic review regarding ophthalmic ADRs to systemic drugs, to systematically summarize evidence and to identify specific areas that lacked systematization or assessment.

- From 562 studies initially found, only 32 were included, and few studies had low risk of bias. Drugs frequently involved included amiodarone, sildenafil, hydroxychloroquine and biphosphonates.

- Many ophthalmic ADRs are frequent but remain unnoticed; therefore, the systematization of specific ophthalmic ADRs, the increase of knowledge and the dissemination of protocols of collaboration are suggested.

\section{ETHICS STATEMENT}

Authors confirm to have adhered to Ethics principles and Helsinqui Declaration during all phases of this study.

\section{ACKNOWLEDGEMENTS}

The authors would like to thank MSD for obtaining the full text of 8 articles and Théa for obtaining the full text of 7 articles. 


\section{REFERENCES}

1. Fraunfelder FW, Fraunfelder FT. Scientific challenges in postmarketing surveillance of ocular adverse drug reactions. Am J Ophthalmol 2007; 143(1): 145-149. Epub 2006 Nov 13.

2. American Academy of Ophthalmology. Fundamentals and principles in basic Ophthalmology. Section 2 in AAO's Basic and Clinical Science Course, Vol. 2, 2012; 249-265.

3. Luykx J, Mason M, Ferrari MD, et al. Are migraineurs at increased risk of adverse drug responses? A meta-analytic comparison of topiramate-related adverse drug reactions in epilepsy and migraine. Clin Pharmacol Ther 2009; 85(3): 283-8. Epub 2008 Nov 5.

4. Fel A, Aslangul E, Le Jeunne C. Eye and corticosteroid's use. Presse Med 2012; 41(4): 414-21.

5. Abdel-Aziz S, Mamalis N. Intraoperative floppy iris syndrome. Curr Opin Ophthalmol 2009; 20(1): 37-41.

6. Cano Parra J, Díaz-Llopis M. Drug induced uveitis. Arch Soc Esp Oftalmol 2005; 80(3): $137-49$

7. Carelli V, Ross-Cisneros FN, Sadun AA. Optic nerve degeneration and mitochondrial dysfunction: genetic and acquired optic neuropathies. Neurochem Int 2002; 40(6): 573-84

8. Hollander DA, Aldave AJ. Drug-induced corneal complications. Curr Opin Ophthalmol 2004; 15(6): 541-8

9. Fraunfelder FT, Edwards R. Possible ocular adverse effects associated with leuprolide injections. JAMA 1995; 273(10): 773-4.

10. Miguel A, Azevedo LF, Araújo M, et al. Frequency of adverse drug reactions in hospitalized patients: a systematic review and meta-analysis. Pharmacoepidemiol Drug Saf 2012; 21(11): 1139-54.

11. Lazarou J, Pomeranz BH, Corey PN. Incidence of adverse drug reactions in hospitalized patients: a meta-analysis of prospective studies. JAMA 1998; 279(15): $1200-5$.

12. Cuervo LG, Clarke M. Balancing benefits and harms in health care. Br Med J 2003; 327(7406): 65-66.

13. Loke YK, Price D, Herxheimer A. Systematic reviews of adverse effects: framework for a structured approach. BMC Med Res Methodol 2007; 7: 32-39.

14. Pirmohamed M, Park BK. Genetic susceptibility to adverse drug reactions. Trends Pharmacol Sci 2001; 22(6): 298-305.

15. Davies EC, Green CF, Mottram DR, et al. Adverse drug reactions in hospitals: a narrative review. Curr Drug Saf 2007; 2: 79-87.

16. Cornelius VR, Perrio MJ, Shakir SAW, et al. Systematic reviews of adverse effects of drug interventions: a survey of their conduct and reporting quality. Pharmacoepidemiol Drug Saf 2009; 18: 1223-1231.

17. Chou R, Fu R, Carson S, et al. Methodological shortcomings predicted lower harm estimates in one of two sets of studies of clinical interventions. $J$ Clin Epidemiol 2007; 60(1): 18-28.

18. Fraunfelder FW. Ocular adverse drug reactions. Expert Opin Drug Saf 2003; 2(4): 411-20.

19. Wren VQ. Ocular \& visual side effects of systemic drugs - clinically relevant Toxicology and patient management. J Beh Optom 2000; 11(6): 149-157.

20. The Cochrane Collaboration. Cochrane Handbook for systematic reviews of interventions version 5.0.0. 2008; 434-449.

21. Moher D, Liberati A, Tetzlaff J, et al. Preferred Reporting Items for Systematic Reviews and Meta-Analyses: The PRISMA Statement. PLoS Med 2009; 6(7): e1000097.

22. World Health Organization. International Drug Monitoring: The Role of the Hospital. World Health Organization: Geneva, Switzerland, 1966. Technical Report Series No. 425.

23. World Health Organization. WHO Draft Guidelines for Adverse Event Reporting and Learning Systems. 2005. Available at: http://www.who.int/patientsafety/ events/05/Reporting_Guidelines.pdf [September 2013].

24. Ferris FL, 3rd, Kassoff A, Bresnick GH, et al. New visual acuity charts for clinical research. Am J Ophthalmol 1982; 94: 91-6.

25. World Health Organization. Anatomical Therapeutic Chemical (ATC) classification system. Available at: http://www.whocc.no/atc/structure and principles/ [October 2013].

26. Naranjo CA, Busto U, Sellers EM, et al. A method for estimating the probability of adverse drug reactions. Clin Pharmacol Ther 1981; 30: 239-245.

27. Hartwig SC, Siegel J, Schneider PJ. Preventability and severity assessment in reporting adverse drug reactions. Am J Hosp Pharm 1992; 49: 2229-32.

28. Schumock GT, Thornton JP. Focusing on the preventability of adverse drug reactions. Hosp Pharm 1992; 27: 538

29. Rawlins MD, Thompson JW. Pathogenesis of adverse drug reactions. In Textbook of adverse drug reactions, Davies DM (ed.). Oxford University Press: Oxford, 1977.

30. Vandenbroucke JP, von Elm E, Altman DG, et al. Strengthening the reporting of observational studies in epidemiology (STROBE): explanation and elaboration. PLoS Med 2007; 4(10): e297.

31. Moher D, Cook DJ, Eastwood S, et al. Improving the quality of reports of metaanalyses of randomized controlled trials: the QUOROM statement. Quality of reporting of meta-analyses. Lancet 1999; 354(9193): 1896-1900.
32. Davidson SI, Rennie IG. Ocular toxicity from systemic drug therapy. An overview of clinically important adverse reactions. Med Toxicol 1986; 1(3): 217-24.

33. Curran CF, Luce JK. Ocular adverse reactions associated with adriamycin (doxorubicin). Am J Ophthalmol 1989; 108(6): 709-11.

34. Hobley A, Lawrenson J. Ocular adverse effects to the therapeutic administration of digoxin. Ophthalmic Physiol Opt 1991; 11(4): 391-3.

35. Malek N, Lebuisson DA. Adverse ocular reactions to oral contraceptive use. Contracept Fertil Sex 1992; 20(4): 441-1.

36. Goldman MJ, Schultz-Ross RA. Adverse ocular effects of anticonvulsants. Psychosomatics $1993 ;$ 34(2): 154-8.

37. Macarol V, Fraunfelder FT. Pamidronate disodium and possible ocular adverse drug reactions. Am J Ophthalmol 1994; 118(2): 220-4.

38. Oshika T. Ocular adverse effects of neuropsychiatric agents. Incidence and management. Drug Saf 1995; 12(4): 256-63.

39. Fraunfelder FW, Fraunfelder FT, Illingworth DR. Adverse ocular effects associated with niacin therapy. Br J Ophthalmol 1995; 79(1): 54-6.

40. Sweeney JA. Adverse effects of risperidone on eye movement activity: A comparison of risperidone and haloperidol in antipsychotic-naive schizophrenic. Neuropsychopharmacology 1997; 16(3): 217-28.

41. Dulley P. Ocular adverse reactions to tamoxifen-a review. Ophthalmic Physiol Opt 1999; 19(Suppl 1): S2-9.

42. Solomon A, Siganos CS, Frucht-Pery J. Adverse ocular effects following influenza vaccination. Eye (Lond) 1999; 13(3a): 381-2.

43. Doughty MJ, Lyle WM. Medications used to prevent migraine headaches and their potential ocular adverse effects. Optom Vis Sci 1995; 72(12): 879-91.

44. Ikaheimo K, Kettunen R, Mantyjarvi M. Adverse ocular effects of flecainide. Acta Ophthalmol Scand 2001; 79(2): 175-6.

45. Fraunfelder FT, Fraunfelder FW, Edwards R. Ocular side effects possibly associated with isotretinoin usage. Am J Ophthalmol 2001; 132(3): 299-305.

46. Ikaheimo K, Kettunen R, Mantyjarvi M. Visual functions and adverse ocular effects in patients with amiodarone medication. Acta Ophthalmol Scand 2002; 80(1): 59-63.

47. Fraunfelder FW, Fraunfelder FT. Adverse ocular drug reactions recently identified by the National Registry of Drug-Induced Ocular Side Effects. Ophthalmology 2004; 111(6): 1248-50.

48. Fraunfelder FW, Solomon J, Mehelas TJ. Ocular adverse effects associated with cyclooxygenase-2 inhibitors. Arch Ophthalmol 2006; 124(2): 277-9.

49. Santaella RM, Fraunfelder FW. Ocular adverse effects associated with systemic medications: Recognition and management. Drugs 2007; 67(1): 75-93.

50. Sowka JW, Neiberg MN, Vollmer LA. Optic atrophy after sildenafil use. Optometry 2007; 78: 122-128.

51. Mandal A, Chatterjee S, Bose S, et al. Ocular adverse effects of Topiramate: Two case reports. Indian J. Pharmacol 2008; 40(6): 278-280.

52. Bell CM, Hatch WV, Fischer HD, et al. Association between tamsulosin and serious ophthalmic adverse events in older men following cataract surgery. JAMA 2009; 301(19): 1991-6.

53. Cordell WH, Maturi RK, Costigan TM, et al. Retinal effects of 6 months of daily use of tadalafil or sildenafil. Arch Ophthalmol 2009; 127(4): 367-73.

54. El-Domyati MM, El-Fakahany HM, Morad KE. Nonarteritic ischaemic optic neuropathy (NAION) after $36 \mathrm{~h}$ of intake of sildenafil citrate: first Egyptian case. Andrologia 2009; 41(5): 319-21.

55. Richa S, Yazbek JC. Ocular Adverse Effects of Common Psychotropic Agents: A Review. CNS Drugs 2010; 24(6): 501-27.

56. Al-Hussaini ZK, McVary KT. Alpha-blockers and intraoperative floppy iris syndrome: ophthalmic adverse events following cataract surgery. Curr Urol Rep 2010; 11(4): 242-8.

57. Lebreton O, Weber M. Ophthalmologic adverse effects of systemic corticosteroids (french). Rev Med Interne 2011; 32: 506-512.

58. Azzouni F, Abu samra F. Are Phosphodiesterase Type 5 Inhibitors Associated with Vision-Threatening Adverse Events? A Critical Analysis and Review of the Literature. J Sex Med 2011; 8(10): 2894-2903.

59. Seitz DP, Campbell RJ, Bell CM, et al. Short-term exposure to antidepressant drugs and risk of acute angle-closure glaucoma among older adults. J Clin Psychopharmacol 2012; 32(3): 403-7.

60. Saint-Jean A, Sainz de la Maza M, Morral M, et al. Ocular adverse events of systemic inhibitors of the epidermal growth factor receptor: report of 5 cases. Ophthalmology 2012; 119(9): 1798-802.

61. Neudorfer M, Goldshtein I, Shamai-Lubovitz O, Chodick G, Dadon Y, Shalev V. Ocular adverse effects of systemic treatment with isotretinoin. Arch Dermatol 2012; 148(7): 803-8.

62. OCEBM Levels of Evidence Working Group. The Oxford Levels of Evidence 2. Oxford Centre for Evidence-Based Medicine. Available at: http://www.cebm. net/index.aspx?o=5653 [26th August 2013]

63. Rosselet E, Faggioni R. Glaucoma and psychotropic drugs. Ophthalmologica 1969; 158(Suppl): 462-8.

64. Garbe E, LeLorier J, Boivin JF, Suissa S. Risk of ocular hypertension or open angle glaucoma in elderly patients on oral glucocorticoids. Lancet 1997; 350: 979-82.

65. Sundmark E. The occurrence of posterior subcapsular cataracts in patients on long-term systemic corticosteroid therapy. Acta Ophthal 1963; 41: 516-23. 